\title{
Front Matter: Volume 9270
}

, "Front Matter: Volume 9270," Proc. SPIE 9270, Optoelectronic Devices and Integration V, 927001 (24 November 2014); doi: 10.1117/12.2178998

SPIE. Event: SPIE/COS Photonics Asia, 2014, Beijing, China 


\title{
Optoelectronic Devices and Integration $V$
}

\author{
Xuping Zhang \\ Hai Ming \\ Changyuan $\mathrm{Yu}$ \\ Editors \\ 9-11 October 2014 \\ Beijing, China \\ Sponsored by \\ SPIE \\ COS_Chinese Optical Society \\ Cooperating Organizations \\ Singapore (Singapore) • European Optical Society \\ Supporting Organizations \\ CAST_China Association for Science and Technology (China) \\ NSFC—National Nature Science Foundation (China) \\ Published by \\ SPIE
}

Tsinghua University (China) - Peking University (China) • University of Science and Technology of China (China) Zhejiang University (China) • Tianjin University (China) • Beijing Institute of Technology (China) • Beijing University of Posts and Telecommunications (China) - Nankai University (China) - Changchun University of Science and Technology (China) • University of Shanghai for Science and Technology (China) • Capital Normal University (China) Huazhong University of Science and Technology (China) • Beijing Jiaotong University (China) • Shanghai Institute of Optics and Fine Mechanics (China) - Changchun Institute of Optics and Fine Mechanics (China) • Institute of Semiconductors (China) • Institute of Optics and Electronics (China) • Institute of Physics (China) • Shanghai Institute of Technical Physics (China) - China Instrument and Control Society (China) • Optoelectronics Technology Committee, COS (China) • SPIE National Committee in China (China) • Optical Society of Japan (Japan) • Optical Society of Korea (Korea, Republic of) - The Australian Optical Society (Australia) • Optics and Photonics Society of

Volume 9270

Proceedings of SPIE 0277-786X, V. 9270

SPIE is an international society advancing an interdisciplinary approach to the science and application of light.

Optoelectronic Devices and Integration V, edited by Xuping Zhang, Hai Ming, Changyuan Yu, Proc. of SPIE Vol. 9270, 927001 - (C) 2014 SPIE · CCC code: 0277-786X/14/\$18

doi: $10.1117 / 12.2178998$

Proc. of SPIE Vol. $9270927001-1$ 
The papers included in this volume were part of the technical conference cited on the cover and title page. Papers were selected and subject to review by the editors and conference program committee. Some conference presentations may not be available for publication. The papers published in these proceedings reflect the work and thoughts of the authors and are published herein as submitted. The publisher is not responsible for the validity of the information or for any outcomes resulting from reliance thereon.

Please use the following format to cite material from this book:

Author(s), "Title of Paper," in Optoelectronic Devices and Integration V, edited by Xuping Zhang, Hai Ming, Changyuan Yu, Proceedings of SPIE Vol. 9270 (SPIE, Bellingham, WA, 2014) Article CID Number.

ISSN: 0277-786X

ISBN: 9781628413434

Published by

SPIE

P.O. Box 10, Bellingham, Washington 98227-0010 USA

Telephone +1 3606763290 (Pacific Time) · Fax +1 3606471445

SPIE.org

Copyright @ 2014, Society of Photo-Optical Instrumentation Engineers.

Copying of material in this book for internal or personal use, or for the internal or personal use of specific clients, beyond the fair use provisions granted by the U.S. Copyright Law is authorized by SPIE subject to payment of copying fees. The Transactional Reporting Service base fee for this volume is $\$ 18.00$ per article (or portion thereof), which should be paid directly to the Copyright Clearance Center (CCC), 222 Rosewood Drive, Danvers, MA 01923. Payment may also be made electronically through CCC Online at copyright.com. Other copying for republication, resale, advertising or promotion, or any form of systematic or multiple reproduction of any material in this book is prohibited except with permission in writing from the publisher. The CCC fee code is 0277-786X/14/\$18.00.

Printed in the United States of America.

Publication of record for individual papers is online in the SPIE Digital Library.

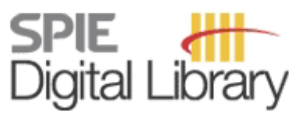

SPIEDigitalLibrary.org

Paper Numbering: Proceedings of SPIE follow an e-First publication model, with papers published first online and then in print and on CD-ROM. Papers are published as they are submitted and meet publication criteria. A unique, consistent, permanent citation identifier (CID) number is assigned to each article at the time of the first publication. Utilization of CIDs allows articles to be fully citable as soon as they are published online, and connects the same identifier to all online, print, and electronic versions of the publication. SPIE uses a six-digit CID article numbering system in which:

- The first four digits correspond to the SPIE volume number.

- The last two digits indicate publication order within the volume using a Base 36 numbering

system employing both numerals and letters. These two-number sets start with 00, 01, 02, 03, 04, $05,06,07,08,09,0 A, 0 B \ldots 0 Z$, followed by 10-1Z, 20-2Z, etc.

The CID Number appears on each page of the manuscript. The complete citation is used on the first page, and an abbreviated version on subsequent pages. Numbers in the index correspond to the last two digits of the six-digit CID Number. 


\title{
Contents
}

\author{
vii Authors \\ ix Symposium Committees \\ xi Conference Committee
}

\section{SESSION 1 OPTOELECTRONIC DEVICES AND APPLICATIONS I}

927004 Characteristic optimization of 1.3- $\mathrm{\mu m}$ InGaAsP MQW lasers for direct modulation applications [9270-3]

927005 Simulation and experiment of 1310nm high speed InGaAsP/InP EAM [9270-4]

927007 Light manipulation for organic light-emitting diodes [9270-6]

$927008 \quad$ 1.2-GHz gated single-photon detector with simple filtering [9270-7]

\section{SESSION 2 OPTOELECTRONIC DEVICES AND APPLICATIONS II}

927009 Effect of surface ligands on the performance of organic light-emitting diodes containing quantum dots [9270-8]

9270 OA Flexible amorphous oxide thin-film transistors on polyimide substrate for AMOLED [9270-9]

9270 OC Electro-optical line cards with multimode polymer waveguides for chip-to-chip interconnects [9270-11]

\section{SESSION 3 LASERS AND AMPLIFIERS}

9270 OF Analysis of wavelength detuning, injected power, and injected mode effect on Fabry-Perot laser diode [9270-15]

9270 OG Experimental investigations on spectrum width of square-wave pulses in passively modelocked figure-8 fiber laser [9270-16]

$9270 \mathrm{OH}$ Performance study on a tunable multiwavelength erbium-doped fiber laser based on nonlinear optical loop mirror and Lyot birefringence fiber filter [9270-17]

92700 Investigations of mode-locked fiber laser based on CVD fabricated graphene saturable absorber [9270-18] 
9270 0J Study on the influence of Mach-Zehnder interferometers to the output characteristics of multiwavelength erbium-doped fiber laser [9270-19]

9270 OK A wavelength conversion circuit for active multi-spectral detection [9270-20]

\section{SESSION 4 SENSORS I}

$9270 \mathrm{OL} \quad$ Performance enhancement for long distance BOTDR sensing system based on high extinction ratio probe pulse generator (Invited Paper) [9270-21]

9270 OM Research of range image on non-scanning LADAR based on APD arrays [9270-22]

9270 ON Compact microfluidic sensing by introducing effective phase shift in fiber Bragg grating [9270-23]

927000 An integral imaging method for depth extraction with lens array in an optical tweezer system [9270-24]

9270 OP Closed-loop experiment of resonator integrated optic gyro with triangular wave phase modulation [9270-25]

\section{SESSION 5 SENSORS II}

9270 OR Wavelength-swept lasers and their application to fiber optic sensors (Invited Paper) [9270-27]

9270 OU Reduced graphene oxide coated optical fiber for methanol and ethanol vapor detection at room temperature [9270-30]

9270 OV A novel fiber Bragg grating wavelength demodulation system based on F-P etalon (Best Student Paper Award) [9270-31]

\section{SESSION 6 OPTICAL COMPONENTS}

9270 OW Electrowetting-based adaptive vari-focal liquid lens array for 3D display (Invited Paper) [9270-32]

9270 0X Wave guiding by low refractive-index strips on surfaces of Chalcogenide glass thin films [9270-33]

9270 OY Birefringence compensated arrayed waveguide grating [9270-34]

$92700 Z$ Photonic drop splitters based on silicon photonic crystal cascaded self-collimation ring resonators [9270-35]

927010 Series-coupled double-ring resonators with asymmetric radii for use in channelizer [9270-36] 
927011 Theoretical investigation of polarization-dependent devices based on the critical guiding condition of SOI waveguide [9270-37]

927012 Aspheric lens based imaging receiver for MIMO visible light communication [9270-38]

927013 Sub-wavelength focusing of cylindrical vector beams by a ID metallic photonic crystal plano-concave lens [9270-39]

\section{SESSION 7 COMMUNICATIONS/SIGNAL PROCESSING}

927014 Excess signal transmission with dimming control pattern in indoor visible light communication systems (Invited Paper) [9270-40]

927016 Optically powered active sensing system for Internet Of Things [9270-42]

927017 Investigation on the BER performance of the MSK space downlink laser communication system with a power EDFA [9270-43]

927018 Optical generation of tunable microwave and millimeter waves by using asymmetric fiber Bragg grating Fabry-Perot cavity fiber laser [9270-44]

$92701 \mathrm{~A}$ Optical properties of $(1-\mathbf{x}) \mathrm{Pb}\left(\mathrm{Zn}_{1 / 3} \mathrm{Nb}_{2 / 3}\right) \mathrm{O}_{3}-\mathrm{xPbTiO}{ }_{3}$ single crystals [9270-73]

\section{POSTER SESSION}

9270 1B Colour tuneable micro-display based on LED matrix [9270-13]

9270 IC Photoemission behaviors of transmission-mode InGaAs photocathode [9270-47]

9270 1D Spin velocity measurement for space debris from periodic signatures with active and passive illumination [9270-48]

9270 1E Passively mode-locked Er-doped fiber laser based on a semiconductor saturable absorber mirror [9270-49]

$9270 \mathrm{lF}$ Theoretical revision of quantum efficiency formula for thin AIGaAs/GaAs photocathodes [9270-50]

9270 IG Efficiency analysis of parallelized wavelet-based FDTD model for simulating high-index optical devices [9270-51]

$92701 \mathrm{H} \quad$ A 3-D optoelectronic integration methodology utilizing CMOS post-backend process [9270-52]

$927011 \quad$ Polymer waveguide based hybrid opto-electric integration technology [9270-53]

$92701 \mathrm{~K}$ Post-digital image processing based on microlens array [9270-55]

9270 IL Micro-cavity lasers with large device size for directional emission [9270-56] 
$92701 \mathrm{M}$ Room temperature ammonia vapor sensing properties of transparent single walled carbon nanotube thin film [9270-57]

927010 Supercontinuum generation in two kinds of chalcogenide microstructured optical fibers [9270-61]

$92701 Q \quad$ Ultra-broadband supercontinuum generation in fluoride glass by filamentation [9270-63]

$92701 \mathrm{~T}$ Assessment of reliability of a code of optical rotary encoders with the serial interface [9270-66]

$92701 \mathrm{~V}$ Mode-locked erbium-doped fiber laser using graphene-covered-microfiber as saturable absorber [9270-68]

$92701 \mathrm{X}$ An effective coding design for improving the performance of LED lighting based optical communication system [9270-70]

9270 IY Thermal influence of phosphor to GaN-based white LEDs [9270-71] 


\title{
Authors
}

Numbers in the index correspond to the last two digits of the six-digit citation identifier (CID) article numbering system used in Proceedings of SPIE. The first four digits reflect the volume number. Base 36 numbering is employed for the last two digits and indicates the order of articles within the volume. Numbers start with 00, 01, 02, 03, 04, 05, 06, 07, 08, 09, OA, OB...0Z, followed by 10-1Z, 20-2Z, etc.

\author{
Cao, Yong, OA \\ Chang, Benkang, 1C \\ Chen, Biao, $0 Z$ \\ Chen, Cong, 18, 1E \\ Chen, Guoliang, OG, Ol \\ Chen, Haiyan, 18, $1 \mathrm{E}$ \\ Chen, Hongda, $1 \mathrm{H}$ \\ Chen, Jian, 14 \\ Chen, Peifeng, OC \\ Chen, Xinlong, 1C \\ Chen, Xi-Yao, $\mathrm{OZ}$ \\ Cheng, Chuantong, $1 \mathrm{H}$ \\ Cheng, Hongchang, $1 \mathrm{C}$ \\ Chistyakov, Alexander, 09 \\ Choi, Byeong Kwon, OR \\ Dayneko, Sergey, 09 \\ Deng, Lingling, 11 \\ Dong, Shandong, 17 \\ Duan, Zhongchao, 10 \\ Feng, Cheng, $1 \mathrm{~F}$ \\ Feng, Lishuang, OP \\ Feng, Yuan, $1 \mathrm{~L}$ \\ Filatov, Yu. V., $1 T$ \\ Fu, Dong, OA \\ Fu, Ping, $\mathrm{OZ}$ \\ Fu, Shenggui, $1 \mathrm{~V}$ \\ Gao, Chen, 16 \\ Gao, Feng, 10 \\ Gao, Weiqing, 10 \\ Gao, Zhuo, OA \\ Gu, Chun, OG \\ Gu, Xiaowen, 10 \\ Guo, Fei, 04 \\ Guo, Jinghong, OV \\ Guo, Ying, OA \\ Han, Shaokun, OK, OM \\ Hao, Yong-gin, $1 \mathrm{~L}$ \\ $\mathrm{He}$, Chongjun, $1 \mathrm{~A}$ \\ He, Jian-Jun, OY \\ Ho, Ho-pui, ON, 11 \\ Hu, Huiling, IA \\ $\mathrm{Hu}$, Jigang, 10 \\ $\mathrm{Hu}$, Yangyang, $\mathrm{Ol}$ \\ Huang, Beiju, $1 \mathrm{H}$ \\ Huang, Kaiqiang, 18, 1E \\ Huang, Yidong, $\mathrm{OX}$ \\ Immonen, Marika, OC \\ Jeon, Min Yong, OR \\ Ji, Chen, 04, 05
}

Jia, Hong, 1 A

Jiang, Jian, 16

Jiang, Jun-Zhen, $\mathrm{OZ}$

Jiang, Xiyan, 1G, 11

Jiao, Wenxiang, 17

Jin, Muchun, $1 \mathrm{C}$

Ju, Qiuqi, 12

Kang, Yanyan, OK, OM

Kavinkumar, T., OU

Ko, Myeong Ock, OR

Kong, Qingfeng, 1B

Kwon, Yong Seok, OR

Lang, Tingting, OY

Li, Baowei, OK, OM

Li, Desheng, $1 X$

Li, Jing, 1B

Li, Jinmin, 1B

Li, Mi, 17

Li, Min, OA

Li, Ming, 1D

Li, Peng, 1L

Li, Qi, 18, 1E

Li, Yan-Qing, 07

Li, Yinmei, 00

$\mathrm{Li}$, Yongfu, 08

$\mathrm{Li}$, Yuan, $\mathrm{OH}, \mathrm{OJ}$

Li, Yuan, 10

Liang, Zhongcheng, 12

Liao, Meisong, 1Q

Lin, Bao-Cheng, $\mathrm{OZ}$

Lin, Jian, OG, Ol

Lin, Yuan-Yuan, $\mathrm{OZ}$

Linkov, Pavel, 09

Liv, Huilan, OP

Liu, Jing-Ping, $0 Z$

Liu, Junliang, 08

Liu, Lili, 1B

Liu, Weiwei, 00

Liu, Xueming, 12

Liu, Youwen, $1 \mathrm{~A}$

Lu, Dan, 04, 05

LU, Pengzhi, 1B, $1 Y$

LU, Yuangang, 17

Lu, Yunqing, $1 \mathrm{G}$

LV, Lidong, OV

Lypenko, Dmitriy, 09

Manivannan, S., OU, IM

Mao, Jinbin, 11

Mao, Qing, 1D 
Mao, Xurui, $1 \mathrm{H}$

Martynov, Igor, 09

Mei, Li, OG, Ol

Ming, Hai, $\mathrm{Ol}, 0 \mathrm{O}$

Nakarmi, Bikash, OF

Namihira, Yoshinori, $1 \mathrm{X}$

Nikolaev, M. S., $1 T$

Ohishi, Yasutake, 10, 1Q

Ou, Qing-Dong, 07

Pan, Shilong, 10

Pang, Jiawei, OA

Pavlov, P. A., IT

Pei, Yanrong, 1B

Peng, Junbiao, OA

Qian, Yunsheng, $1 \mathrm{~F}$

Qiu, Huaili, 10

Quan, Mingran, $\mathrm{OH}, \mathrm{OJ}$

Rapala-Virtanen, Tarja, OC

Ren, Rong, 1G, 11

Samokhvalov, Pavel, 09

Sastikumar, D., OU

Shi, Chaiyuan, IK

Shi, Jian-wei, $1 \mathrm{~L}$

Shi, Ruizhi, OC

Shobin, L. R., $1 M$

Song, Yuejiang, 17

Sun, Biao, OG

Suzuki, Takenobu, 10

Tameev, Alexey, 09

Tang, Jian-Xin, 07

Tang, Minghui, ON

Tang, Yichuang, $\mathrm{OP}$

Tian, Jiajun, $\mathrm{OH}, \mathrm{OJ}$

Tu, Guojie, OV

Wan, Hongdan, 16

Wang, Anting, OG, 00

Wang, Chunfu, OA

Wang, Guanghui, OL, ON, 11

Wang, Huitao, 05

Wang, Jiming, $1 \mathrm{~A}$

Wang, Jin, 12, 13, 16, 1G, 11

Wang, Junjie, OP

Wang, Junxi, 1B, $1 Y$

Wang, Lei, OA

Wang, Liangliang, 1D

Wang, Meng, 18, 1E

Wang, Shulu, 00

Wang, We, 04

Wang, Xiaotong, 1B

Wang, Zuqiang, 08

Won, Yong Hyub, OW

Wu, Jinhua, OC

Wu, Xuelin, OL

Xia, Lan, OL, OV

Xia, Wenze, OK, OM

Xia, Xiang, OY

Xie, Haizhong, 1B

$\mathrm{XU}$, Feng, $1 \mathrm{~K}$

$X \cup$, Guoliang, OV

$\mathrm{XU}, \mathrm{Ji}, 13,1 \mathrm{G}$
$\mathrm{XU}$, Lixin, OG, OI

$X \cup, M i a o, O A$

$X \cup$, Zhiping, $O A$

Xue, Bin, $1 \mathrm{~B}$

Xue, Li, 1D

Yan, Chang-ling, $1 \mathrm{~L}$

Yan, Hui Juan, OC

Yang, Gang, OV

Yang, Hua, 1B

Yang, Jing, 16

Yang, Li-Hui, $\mathrm{OZ}$

Yang, $\mathrm{Na}, \mathrm{IX}$

Yang, Tingting, 12

Yang, $Y \mathrm{i}, \mathrm{IX}$

Yao, Yong, $\mathrm{OH}, \mathrm{OJ}$

Yi, Xiaoyan, 1B

Yin, Long, 16

Ying, Zhoufeng, 11

You, Xiaodi, 14

Yu, Changyuan, 14

Yu, Fei, 1B

Yuan, Zijun, 10

Zeng, Y. P., $1 Y$

Zhai, Yanfen, OX

Zhai, Yumeng, 11

Zhang, Chunfang, 08

Zhang, Dongying, OF

Zhang, Ruikang, 04, 05

Zhang, Wei, OX

Zhang, Xianming, OG

Zhang, Xuping, OF, OL, ON, 11, 17

Zhang, Yijun, 1C, IF

Zhang, Yixin, OL

Zhang, Yuncui, $1 \mathrm{X}$

Zhang, Zan, $1 \mathrm{H}$

Zhang, Zanyun, $1 \mathrm{H}$

Zhao, L. X., IY

Zhao, Yongjiu, 10

Zheng, H. W., $1 Y$

Zheng, Huanhuan, 14

Zhi, Yinzhou, OP

Zhong, Yi, 13

Zhou, Bojun, IV

Zhou, Lei, 07

Zhou, Lei, OA

Zhou, Z. C., IY

Zhu, Dan, 10

Zhu, Dongda, $1 \mathrm{~L}$

Zhu, Kongjun, 1A

Zhu, Long Xiu, OC

Zou, Jianhua, OA

Zou, Jun, OY

Zou, Nianyu, $1 \mathrm{X}$ 


\title{
Symposium Committees
}

\author{
General Chairs
}

H. Philip Stahl, NASA Marshall Space Flight Center (USA)

Bingkun Zhou, Tsinghua University (China)

General Co-chairs

Arthur Chiou, National Yang-Ming University (Taiwan, China)

Jianlin Cao, China Ministry of Science and Technology (China)

Junhao Chu, Shanghai Institute of Technical Physics (China)

Technical Program Chairs

Songlin Zhuang, Shanghai University of Science and Technology (China)

Xingde Li, Johns Hopkins University (United States)

Technical Program Co-chairs

Qiming Wang, Institute of Semiconductors (China)

Xu Liu, Zhejiang University (China)

Daoyin Yu, Tianjin University (China)

Qihuang Gong, Peking University (China)

Tianchu Li, National Institute of Metrology (China)

Wei Huang, Nanjing University of Posts and Telecommunications (China)

Local Organizing Committee Chair

Guangcan Guo, University of Science and Technology of China (China) 
Local Organizing Committee Co-chairs

Guoqiang Ni, Beijing Institute of Technology (China)

Shusen Xie, Fujian Normal University (China)

Xiaomin Ren, Beijing University of Posts and Telecommunications (China)

Ying Gu, People's Liberation Army General Hospital (China)

Huilin Jiang, Changchun University of Science and Technology (China)

General Secretary

Qihuang Gong, Peking University (China)

Local Organizing Committee

Yan Li, Chinese Optical Society/Peking University (China)

Zhiping Zhou, Peking University (China)

Changhe Zhou, Shanghai Institute of Optics and Fine Mechanics (China)

Qingming Luo, Huazhong University of Science and Technology (China)

Chongxiu Yu, Beijing University of Posts and Telecommunications (China)

Hongda Chen, Institute of Semiconductors (China)

Yongtian Wang, Beijing Institute of Technology (China)

Yiping Cui, Southeast University (China)

Xuping Zhang, Nanjing University (China)

Feijun Song, Daheng Corporation (China)

Cunlin Zhang, Capital Normal University (China)

Yanting Lu, Nanjing University (China)

Yuejin Zhao, Beijing Institute of Technology (China)

Chunqing Gao, Beijing Institute of Technology (China)

Tiegen Liu, Tianjin University (China)

Xiaocong Yuan, Nankai University (China)

Weimin Chen, Chongqing University (China)

Zhongwei Fan, Academy of Optoelectronics (China)

Hanyi Zhang, Tsinghua University (China)

Lan Wu, Zhejiang University (China)

Yongsheng Zhang, University of Science and Technology of China (China)

Hong Yang, Peking University (China)

Xiaoying Li, Tianjin University (China)

Wei Xiong, Chinese Optical Society (China) 


\title{
Conference Committee
}

\author{
Conference Chairs
}

Xuping Zhang, Nanjing University (China)

Hai Ming, University of Science and Technology of China (China)

Changyuan Yu, National University of Singapore (Singapore)

\author{
Conference Program Committee \\ Dayan Ban, University of Waterloo (Canada) \\ Seth Bank, The University of Texas at Austin (United States) \\ Zhongping Chen, Beckman Laser Institute and Medical Clinic \\ (United States) \\ Ho-Pui A. Ho, The Chinese University of Hong Kong \\ (Hong Kong, China) \\ Jan Ingenhoff, lonexphotonics Inc. (Canada) \\ Zhong-cheng Liang, Nanjing University of Posts and \\ Telecommunications (China) \\ Xuejun Lu, University of Massachusetts Lowell (United States) \\ Bikash Nakarmi, Korea Advanced Institute of Science and \\ Technology (Korea, Republic of) \\ Gang-Ding Peng, The University of New South Wales (Australia) \\ Yuan Shi, Agilecom Photonic Solutions Inc. (United States) \\ Yuejiang Song, Nanjing University (China) \\ Anna K. Swan, Boston University (United States) \\ Frank Vollmer, Max-Planck-Institut für die Physik des Lichts (Germany) \\ Guanghui Wang, The Chinese University of Hong Kong \\ (Hong Kong, China) \\ Daniel M. Wasserman, University of Illinois at Urbana-Champaign \\ (United States) \\ Lixin Xu, University of Science and Technology of China (China)
}

\section{Session Chairs}

1 Optoelectronic Devices and Applications I

Xuping Zhang, Nanjing University (China)

2 Optoelectronic Devices and Applications II

Xuping Zhang, Nanjing University (China)

3 Lasers and Amplifiers

Hai Ming, University of Science and Technology of China (China) 
4 Sensors 1

Weiqing Gao, Hefei University of Technology (China)

5 Sensors II

Jian Chen, Nanjing University of Posts and Telecommunications (China)

6 Optical Components

Jian Chen, Nanjing University of Posts and Telecommunications (China)

7 Communications/Signal Processing

Bikash Nakarmi, Korea Advanced Institute of Science and Technology (Korea, Republic of) 